\title{
Historia y aspectos sociales de la alergología en Cuba
}

\author{
History and social aspects of allergy in Cuba
}

\author{
Magaly del Pilar Olivares Elegia,' Marisela Pérez Pacareu," Rosa María \\ Naranjo Robalino'"I
}

' Doctor en Medicina. Especialista de II Grado en Alergología. Máster en Infectología. Hospital Universitario "Dr. Miguel Enríquez". La Habana, Cuba.

"Doctor en Medicina. Especialista de II Grado en Alergología Máster en Longevidad Satisfactoria. Hospital Universitario "Dr. Gustavo Aldereguía Lima". Cienfuegos, Cuba.

III Doctor en Medicina. Especialista de II Grado en Alergología. Máster en Infectología. Hospital Clínicoquirúrgico "Joaquín Albarrán". La Habana, Cuba.

\section{RESUMEN}

La especialidad de alergología compite entre les más caras de la práctica médica en los países desarrollados, y casi entre las ausentes en los países subdesarrollados, por lo que una gran parte de la población queda sin atención promoviendo el criterio de que la alergia es una enfermedad de ricos y que los pobres no la padecen, cuando en realidad se trata de un problema de posibilidades de acceso a estos servicios. Se presenta un bosquejo históricosocial de la especialidad en general y de Cuba en particular, donde la alergología tiene una organización social diferente comparada con otros países. Se hace un enfoque de la filosofía humanista en que está basada la especialidad y referencia al esfuerzo que han hecho y hacen los científicos para mantener bien bajas las tasas de morbilidad y mortalidad de estas enfermedades en Cuba a la altura de los países desarrollados. La especialidad de alergología en Cuba ha tenido un salto cualitativo ya que tienen acceso todos sus ciudadanos sin tener en cuenta raza, condición social, económica, política o religiosa.

Palabras clave: Alergia, historia, estructura social. 


\title{
Revista Cubana de Salud Pública. 2011;37(3):338-348
}

\begin{abstract}
The allergy specialty is one of the most expensive disciplines in the medical practice in the developed countries, and one of the specialties almost absent in the underdeveloped countries. This is the reason why large sections of the population are left unattended and it is stated that allergy is a disease of rich people, the poor people do not suffer from dermal diseases; however, the realities show that the problem has to do with access to these services. A historical and social outline of this specialty in general and particularly in Cuba, where allergy exhibits different social organization compared with other countries, was presented. It focused on the humanist philosophy that supports this specialty and stated the efforts by the Cuban scientists to keep very low morbidity and mortality rates from these diseases in Cuba, comparable to those of the developed countries. The allergy specialty has increased its qualitative level since all the Cuban citizens get access to this service regardless of race, social status, economic conditions, political ideas or religious beliefs.
\end{abstract}

Key words: allergy, history, social structure.

A los Drs. Rubén Rodríguez Gavaldá, Javier Fernández de Castro y Armando O. Gómez Echevarria, in memorian

\section{NTRODUCCI ÓN}

La especialidad de alergología en todos los países es minoritaria en relación con otras especialidades, "anémica" diría el profesor Gavaldá y esto no es debido precisamente a que existan pocos pacientes alérgicos, sino a que aparentemente no es costeable para toda la población desde el punto de vista económico. Mucho más si los servicios de salud son privados, por tal motivo enfermedades clasificadas como crónicas no transmisibles aumentan por falta de prevención, de una intervención a tiempo, de un consejo genético o de una rehabilitación oportuna. Los países en vías de desarrollo son los más afectados. En Cuba la organización de la salud es gubernamental dirigida por el Ministerio de Salud Pública (MINSAP) en la que se contempla también la especialidad de alergología, que lleva sus servicios hasta el nivel primario de salud, interviene precozmente incluso con inmunoterapia, único método reconocido por la OMS como capaz de cambiar el curso natural de la enfermedad, previniendo de esta forma secuelas invalidantes y aumentando la calidad de vida de los pacientes. Por tal motivo, los profesionales que integran el sistema de salud no escapan de ese empeño y a pesar de que en ocasiones, los métodos, instrumentos de investigaciones y tratamientos se ven afectados por problemas políticos y económicos a causa de un arcaico bloqueo que dura más de 50 años, las bases científicas de la formación están creadas para sortear tales obstáculos y salir adelante a pesar de las dificultades. No se encontraron antecedentes sobre el desarrollo de la alergología en Cuba y es de interés profundizar en el tema porque ayuda a esclarecer la organización de salud en el contexto social cubano y recoge experiencias de cómo llevar salud para todos. El propósito planteados al respecto es hacer un bosquejo históricosocial de la especialidad de alergología en Cuba. 


\section{MÉTODOS}

Revisión de la literatura médica y social al respecto. Comparación de materiales teóricos e informativos de diferentes fuentes bibliográficas y su organización cronológica.

\section{BOSQUEJ O HISTÓRI COSOCI AL DE LA ESPECI ALI DAD DE ALERGOLOGÍ A}

La alergología es la especialidad médica que estudia a pacientes con enfermedades alérgicas. ¿Y que es la alergia? No es más que una reacción de hipersensibilidad, iniciada por mecanismos inmunológicos y mediados por células o anticuerpos. ${ }^{1,2}$ No es lo mismo que atopia que es una predisposición genética con una hipersensibilidad mediada por IgE a alergenos comunes que no son nocivos a la mayoría de las personas. ${ }^{1,3,4}$

La alergia no surge como ciencia espontáneamente sino como resultado de fenómenos no esperados en el campo investigativo de la inmunología, por lo tanto la consideramos parte de esta especialidad aunque tiene sus especificidades. Los primeros artículos de inmunología desde el punto de vista científico, nacen en 1796 con la exitosa vacunación contra la viruela, luego de largos estudios sobre la cow-pox (viruela de las vacas), llevada acabo por Edward J enner, el cual inoculó en el brazo de un joven material de la pústula proveniente de una granjera contaminada, al cabo de varios días el joven presentó una pústula en el área de la vacunación que se curó por sí sola y demostró que este no era afectado por la enfermedad. ${ }^{5}$

Un siglo después Luís Pasteur estudió los trabajos de Jenner y comenzó a inyectar microorganismos debilitados en animales, así descubrió una vacuna para el cólera de las aves y el ántrax del ganado y la rabia, confirmando la posibilidad de proteger contra las infecciones mediante cepas atenuadas de microorganismos patógenos y a finales del siglo XX ya existían vacunas para unas veinte enfermedades. ${ }^{5,6}$

Aunque desde el punto de vista social en ocasiones su generalización era difícil, por ejemplo, tuvieron que pasar 182 años desde el descubrimiento de Jenner para que la OMS declarara la erradicación de la enfermedad a nivel mundial. ${ }^{7}$ Con la introducción de la vacunación como un método efectivo para combatir las enfermedades infecciosas, se desata una escalada en la fabricación de diferentes tipos de vacunas para otros tipos enfermedades inclusive no infecciosas.

Pronto empezaron aparecer reacciones no esperadas con la inmunización y aunque se tenía información sobre procesos alérgicos desde la antigüedad, como el recogido en el jeroglífico de Menfis de una reacción alérgica que data del año 264 A.C. debido a la picadura de una abeja al faraón Menes, quien muere debido a una reacción anafiláctica, no se tenía clara su naturaleza. ${ }^{5}$

En 1901 el príncipe de Mónaco Alberto I, al tanto de los últimos adelantos de la medicina e interesado en cuestiones de inmunidad le pide al famoso investigador Charles Richet que estudiara el efecto tóxico de cierta fisalia a bordo de su yate particular el cual estaba equipado con un moderno laboratorio, el experimento decisivo fue realizado en el perro Neptuno, al intentar una protección con el extracto de una toxina procedente de la anémona sulfata. En la segunda dosis administrada, que se suponía produjera inmunidad, el perro presenta una reacción con disnea, vómitos, diarreas y muerte, a lo cual denominaron anafilaxis por considerarla erróneamente contraria a la profilaxis. Sin embargo aunque el resultado de la 


\section{Revista Cubana de Salud Pública. 2011;37(3):338-348}

investigación fue inesperado y contrario a lo que se esperaba, le valió a Richet recibir el premio Nóbel en 1913 por considerarse que abrió las puertas a nuevos conocimientos en los fenómenos de la inmunología y la alergia. ${ }^{5}$

En 1906 Clement Von Pirquet crea el término de alergia como una reactividad alterada del sistema inmunitario, y ya en 1911 Noon y otros comienzan a vacunar a pacientes alérgicos al polen con buenos resultados. ${ }^{8}$ Se inicia una nueva era en la alergología desde el punto de vista social.

Desafortunadamente los grandes avances que se han producido en esta especialidad no han contribuido a llevar el bienestar social a casi el $40 \%$ de la población mundial que se considera alérgica actualmente y ha funcionado en favor de intereses privados, que han hecho un negocio de sus avances científicos y técnicos, mientras la gran población especialmente la de los países subdesarrollados, carece de atención médica, incluso en enfermedades mucho más urgentes y más fáciles de erradicar.

Esto no tiene que ver necesariamente con el crecimiento económico del país, sino con una voluntad política y de la distribución de las riquezas. El desarrollo de los servicios de salud no depende solo de los recursos financieros. Hay que conocer que en cada momento y lugar el ser humano es el problema central, no el dinero, los programas o la tecnología. Es necesario actuar sistemáticamente sosteniendo que el paciente es lo primero. ${ }^{9}$ Para que esto sea posible es necesario tener en cuenta que la equidad en salud significa iguales oportunidades de acceso a los recursos disponibles, una distribución democrática del poder y de los conocimientos en el sistema de salud, una política de salud que beneficie a todos sin consentir privilegios debido a diferencias de raza, género, territorio, discapacidad u otro rasgo de distintiva grupal o personal. ${ }^{9,10}$ La equidad en salud tiene muchos aspectos y lo mejor es verla como un concepto multidimensional, incluye aspectos relacionados con el logro de la salud y la posibilidad de tener buena salud, y no sólo con la distribución de la atención sanitaria. ${ }^{10}$

En general, el desarrollo alcanzado por la industria farmacéutica transnacional de los países desarrollados de economías de mercado obtiene ganancias gigantescas con la comercialización de productos cuyos costos de producción son bajísimos. Esto explica que muchos de esos medicamentos no pueden estar al alcance de todos los que lo necesitan, y sean además un factor importante en la fuga de divisas de los países subdesarrollados; muchos de estos medicamentos y vacunas, podrían producirse a muy bajo costo en los países del tercer mundo. ${ }^{11}$

Esta forma de distribución de la salud en el mundo capitalista hace que las estadísticas reflejen solo una parte de las sociedades y que otras sean totalmente ignoradas, falseando los resultados de diferentes investigaciones.

En ese sistema de salud donde el dinero es fundamental para recibir atención médica, se abusa por algunos médicos de exámenes sofisticados que incluso pueden provocar complicaciones al paciente, con el único fin de obtener ganancias en detrimento del humanismo y la solidaridad que deben definir a un médico, lo que hace que el paciente pierda la confianza en quien lo atiende. Se obvian aspectos preventivos que pudieran evitar el empeoramiento e incluso la aparición de muchas enfermedades, ${ }^{12}$ como sería la inmunoterapia en fase temprana, único tratamiento capaz de cambiar el curso de la enfermedad. ${ }^{1,13}$ 


\section{DE LA CONSULTA PRI VADA A LA PROYECCI ÓN COMUNITARI A EN CUBA}

\section{I nicios de la Sociedad Cubana de Alergología}

Un mismo término puede usarse para designar posturas filosóficas diferentes en dependencia del país, por ejemplo, mientras el derecho a la salud que en Cuba es un derecho constitucional, jurídico legal y éticomoral, en muchos países, especialmente en los subdesarrollados, todavía se hacen grandes esfuerzos por incorporarlo a la constitución argumentando el mismo principio moral en que se fundamenta los derechos humanos, es decir, el derecho igualitario que merecen todas las personas. Estos hechos sitúan a Cuba en una posición filosófica privilegiada comparada con la de los países tanto del tercer mundo como a la de los de países desarrollados. ${ }^{14,15}$

La prevalencia de las enfermedades alérgicas y del asma en particular han ido aumentando progresivamente y se estima que actualmente el $40 \%$ de la población mundial es alérgica, y para el 2020 se pronostica que lo sean la mitad de los habitantes del planeta, el asma, paradigma de enfermedad alérgica es la principal causa de hospitalización en el mundo occidental especialmente en los niños, por tales motivo la alergia ha sido catalogada por algunos como la epidemia no infecciosa del siglo XXI. ${ }^{16-18}$

Cuba exhibe actualmente en materia de salud estadísticas muy similares a la de los países desarrollados pero con un enfoque social diferente.

Antes de 1959 la alergología en Cuba seguía los mismos cánones que otros países capitalistas, organizada en unas cuantas consultas privadas en la capital del país con altos precios para poder acceder a ellas. Este trabajo está a cargo de profesionales que habían logrado su adiestramiento o especialización en el extranjero. Algunas clínicas privadas y hospitales estatales cuentan con servicios de alergia pero son absolutamente insuficientes para satisfacer las necesidades de la población. Los alergenos y vacunas se adquieren en algunos laboratorios nacionales o importados de los Estados Unidos y se emplean en la práctica privada de la especialidad. ${ }^{6}$

Por esa época, solo practicaban la especialidad una decena de médicos y sin embargo, ya la alergología en Cuba estaba caracterizada como una especialidad, lo que se demuestra por el hecho de que el 16 de octubre de 1947 se reunieron los Drs. Fermín Alpizar, J osefina Amiguet, J osé Cadrechea, Gonzalo Estrada de la Riva, Javier Fernández Castro, José Pedrera, J osé M. Quintero y Julio de los Santos, para aprobar los estatutos de la Sociedad Cubana de Alergia. ${ }^{6,19}$

El 7 de abril de 1948 hicieron la solicitud de creación de la sociedad ante el Gobierno Provincial, el 12 del propio mes les respondieron que la solicitud había sido aprobada.

El 26 de agosto de 1948 queda legalmente constituida la Sociedad Cubana de Alergia, presidida desde ese momento el Dr. Estrada de la Riva hasta 1958. El 6 de noviembre de 1948 por Decreto del Gobierno Provincial fue aprobada e inscrita la sociedad en el Registro Especial de Asociaciones, Libro 23, Folio 202, No. 14632.19

En esta etapa del desarrollo de la alergología es obvio que la docencia oficial no tuviera lugar alguno, solo estaba representada por cursos de posgrado y la proyección futura se perfilaba hacia una mayor privatización y selección elitista tanto de médicos como de pacientes. ${ }^{6,19}$ 


\section{Revista Cubana de Salud Pública. 2011;37(3):338-348}

\section{Primeros laboratorios públicos de alergología}

Dado lo difícil que resultaba estandarizar las vacunas a nivel institucional o nacional comienzan a producirse en laboratorios asociados a consultas privadas de alergia que trabajan en favor de sus propios intereses. Esta poca disponibilidad de recursos costosos y la especificidad que requieren dichas vacunas trajo como consecuencia que las consultas de alergia resultaran ser unas de las más costosas de la práctica médica. Del mismo modo, la complejidad de sus métodos investigativos, la peligrosidad potencial que los rodeaba así como la necesidad de un personal adiestrado y calificado encarecían dicha consulta, esto dio lugar a que se conformara el criterio de que las enfermedades alérgicas eran "enfermedades de ricos", que los pobres no tenían alergias, aunque lo que realmente no tenían era el dinero para tratarse.

Después del triunfo de la revolución en los primeros años de la década del 60 los pocos servicios de alergología apenas podían satisfacer las necesidades de la población dese la óptica asistencial y no existían programas de investigación ni de prevención. Se comienzan los estudios para producir en los laboratorios vacunas a partir del polvo de casa. En mayo de ese año se ordena la gratuidad de los servicios médicos, en julio se nacionalizan los laboratorios farmacéuticos y en agosto se promulga la ley que declara el carácter socialista de la Salud Publica y se designa al MINSAP como rector de todas las actividades sanitarias del país. ${ }^{20}$

A partir del año 1961 se orienta la creación de los servicios de alergia en los hospitales que brindan servicios a la población y el Dr. Armando Gómez Hechevarria es responsabilizado para iniciarlo en el Hospital Universitario "Calixto García" junto con la Dra. Mirtha Sordo en la preparación de extractos alergénicos y la enfermera Inés Cordero en la dilución para la preparación de vacunas, todos procedentes del Consejo Corporativo. ${ }^{19}$

En 1962 se inicia como técnica de alergia Coralia Cainzos Brito y en 1963 Marcos Tulio Chapotin, que pasan a la producción de extractos alergénicos para distribuirlos a los diversos servicios del país. ${ }^{19}$

Posteriormente en 1964 Marta Fernández Menéndez se incorpora como técnica para realizar las pruebas cutáneas. ${ }^{19}$

Se producen extractos de polvo domestico, hongos anemófilos, alimentos, bacterias, pólenes e insectos, que se utilizan para pruebas cutáneas y para vacunas.

Por su parte, el Dr. Rubén Rodríguez Gavaldá, en la misma época, organiza el servicio y laboratorio de alergia e inmunología en el Hospital Pediátrico "William Soler" y se acuerda producir extractos en ese y en el Hospital Universitario "Calixto García" para distribuirlos nacionalmente. Estos fueron los primeros pasos para hacer que la alergología estuviera al alcance de todos. ${ }^{19}$

Los especialistas de ambos hospitales comienzan a producir alergenos y vacunas, especialmente de polvo de casa, de modo que solucionan la situación creada con un ahorro considerable de divisas al país. La tecnología disponible se le entrega al laboratorio "Mario Muñoz". ${ }^{6}$ El 14 agosto 1992 se funda el Centro Nacional de Biopreparados (BIOCEN) y en el departamento de alergenos de dicha institución se comienzan rápidamente las investigaciones y el desarrollo de productos alergénicos para diagnóstico e inmunoterapia de la alergia. ${ }^{21,22}$

En el 2002 recibe se aprueba la primera Regulación Nacional sobre registro de productos alergénicos por el Centro Estatal para el Control de la Calidad de los Medicamenstos (CECMED) y en el 2005 se registra y patenta como vacuna terapéutica 


\section{Revista Cubana de Salud Pública. 2011;37(3):338-348}

subcutánea el producto "Valergen" de comprobada eficacia y seguridad, para varios alergenos. ${ }^{23,24}$

\section{Formación de nuevos alergólogos y socialización de la especialidad}

¿Es la alergia una enfermedad de ricos?

Después del triunfo de la Revolución en 1959 la mayoría del personal médico burgués emigró a los Estados Unidos, lo que marca un antes y un después de esta fecha ya que quedaron en el país solo seis médicos de profesión y de vocación con deseos de llevar la alergología a todos los rincones de la isla, de estos médicos, dos de ellos aún con avanzada edad se mantienen activos en la docencia y son el orgullo de sus discípulos.

Alrededor del año 1970 se inicia la docencia en los Hospitales "Calixto García" y "William Soler", lo que permitió que el 4 de mayo de 1973 el país contara, por primera vez en la historia de la medicina en Cuba, con 6 especialistas graduados de alergología en el país ${ }^{25}$ y en 1984 se incorpora el Hospital Clínicoquirúrgico "Hermanos Ameijeiras" y ya en 1986 aumenta el número de de alergólogos a 103 y más de 250 en el $2006 .{ }^{19}$ Se multiplicaron las consultas en todo el territorio y hoy hay especialistas en todas las provincias con sus correspondientes laboratorios y el gobierno está enfrascado en una labor ardua pero muy humana que es llevar la alergología a todos los municipios del país mediante la proyección comunitaria de la atención primaria de salud (APS), que ya tiene resultados de trabajo en muchos lugares. La cobertura de atención de los pacientes alérgicos en Cuba es total y completamente gratuita, hecho único a nivel mundial. En estas consultas tienen especial importancia los aspectos preventivos, el tratamiento intercrisis, la consejería genética, el embarazo, la lactancia materna, y la inmunoterapia, entre otros. Un lugar muy especial ocupa la escuela para niños asmáticos y familiares, que tiene sus premisas en los campamentos de veranos y en la escuela para niños con grados severos de la enfermedad que cursaban sus estudios internados en esta institución a la orilla del mar. Se enfatiza en el control ambiental, y se profundiza en las alergias ocupacionales, así como en garantizarles a los pacientes una atención especializada una vez establecida la enfermedad y mejorarles su calidad de vida mediante la rehabilitación una vez determinados los daños estructurales. Total acuerdo con Munthel cuando dice que la profesión médica no es un comercio ni tráfico del sufrimiento sino un servicio a la comunidad y un modo honesto de ganarse la vida. ${ }^{26} \mathrm{Y}$ la autora añade: una acción muy humana y gratificante para el médico.

En 1974, se materializa el Programa Nacional de Atención al Asmático. La aplicación de este programa significa el entrenamiento de centenares de médicos, técnicos y enfermeras así como la introducción de medicamentos preventivos que tienen gran impacto de salud en la sociedad como el Cromoglicato de Sodio por inhalación que se aplica es ese momento a todos los alumnos de la enseñanza media y mejora rápidamente los indicadores de salud respiratoria. ${ }^{6,19}$ En 1983 el asma bronquial tiene una prevalencia nacional de $8,33 \%$, pero en 1993, año de férreo bloqueo, los indicadores epidemiológicos aumentan, por lo que en esa década se revitaliza el Programa y se observa la recuperación de algunos de estos indicadores. ${ }^{19}$

A partir del año 1999, el asma bronquial como prototipo de las enfermedades alérgicas ha experimentado un aumento de la dispensarización (control por el médico de la familia) en la APS. En el 2004 constituye la segunda enfermedad de mayor prevalencia de dispensarizados (87,4 × 1000 habitantes, cifra que se incrementa en el $1,4 \%$ en comparación con el 2003). Representa la primera causa de egresos hospitalarios dentro de las enfermedades no transmisibles. En el año 2004 el riesgo de morir por esta causa es de 2,4 x 100000 habitantes, y aunque es baja, la 


\section{Revista Cubana de Salud Pública. 2011;37(3):338-348}

mortalidad extrahospitalaria continúa oscilando entre el 50 y el $64 \%$, más elevada en las ciudades, cuyas causas fundamentales estriban en subestimar los síntomas tanto por parte del paciente como en algunos casos por los médicos. ${ }^{19}$

Algunos de los propósitos del MINSAP para el 2015 son los siguientes: disminuir en el $10 \%$ la mortalidad por asma bronquial, y en el $45 \%$ la mortalidad extrahospitalaria, entre otros. ${ }^{19}$

\section{EL DESARROLLO CI ENTÍ FI COTÉCNI CO Y LA RELACI ON MÉDI CO PACI ENTE}

La industrialización acelerada, la migración a las grandes ciudades y la centralización e institucionalización de los servicios médicos trajo como consecuencia el alejamiento del paciente y su familia y de los servicios primarios o más cercanos de salud. La comunicación médico-paciente se redujo notablemente y el profesional comienza a ser influido por la ola creciente de tecnificación. La concepción del hombre involuciona hasta asumirse como "un conjunto de órganos", el objetivo médico es otra vez tratar enfermedades y no enfermos. ${ }^{27}$

La alergología como el resto de las ramas de la medicina descansa en un principio francamente humanista al reconocer al hombre como valor supremo. En la especialidad se valora altamente la relación médico-paciente ya que muchas de las enfermedades están enmarcadas en las categorías de psicosomáticas, se benefician en gran medida por el arte del médico para llegar a lo profundo de la psiquis y se complementa con la tecnología actualizada.

En esta etapa de alto tecnicismo, se produce la primera gran crisis de humanismo de la profesión médica, proliferan las súper especialidades, y en el trayecto hacia lo multidisciplinario se quiso prescindir del clínico coordinador e integrador, por lo que la relación profesional y despersonalizada, resulta sepultada por toneladas de papeles con gráficas e informes. ${ }^{27}$

Es la intención de los profesores llevar la enseñanza a sus alumnos que sin prescindir de la técnica no deben olvidar jamás la esencia clínica y humanista de la profesión.

Las enfermedades alérgicas constituyen un problema médico social ya que afecta aproximadamente un tercio de toda la isla, por lo tanto el principal objetivo como especialidad estaría encaminado a disminuir su morbilidad y mortalidad, garantizar la calidad de la atención médica, tanto en aspectos preventivos como curativos, fundamentalmente en la atención primaria donde se hacen los máximos esfuerzos, en la atención secundaria cuando ya la enfermedad está establecida, y en su rehabilitación en caso de daños mayores. Todos estos aspectos en la sociedad cubana se inscriben en un sustrato filosófico en cuyo concepto la calidad de la atención médica está dada por dos dimensiones principales: el de la relaciones humanas cuya máxima expresión es la relación médico-paciente, y el de la dimensión técnica que consiste en la aplicación de la ciencia y la tecnología médica de modo que registre el máximo de beneficio a la salud del paciente con un mínimo de riesgo y ofrezca la mejor atención con los recursos disponibles. Tanto la dimensión técnica como la interpersonal tienen fundamentos científicos y no deben primar una sobre la otra. ${ }^{28}$

En Cuba se obtienen buenos resultados a pesar de las grandes dificultades que enfrentamos porque la ciencia, no es la obra de un individuo aislado, sino una actividad profesional institucionalizada que se traduce en un beneficio social. ${ }^{29}$ 
Partiendo del hecho de que la atención médica que se necesita requiere de un alto nivel científico técnico se hace imprescindible la calificación y la constante superación del personal de la salud. Esto es difícil en algunos países, ya que más que recursos, se necesita una política de desarrollo científico que haga que estos conocimientos y técnicas estén disponibles para la mayoría, elementos contemplados en el sistema de salud cubano y también en la especialidad de alergología.

La globalización de la economía repercute en los países subdesarrollados así como el neoliberalismo, que elimina la responsabilidad del estado en la salud pública transformando al individuo en consumidor solamente y a las naciones en mercados, causando perjuicios de toda índole entre los trabajadores científicos que pueden abandonar sus planes de desarrollo independientes e integrarse a la emigración, fundamentalmente hacia las antiguas metrópolis. ${ }^{30}$

Es tarea de todo científico cubano no dejar que por motivos de bloqueo de cualquier índole las investigaciones científicas, principalmente las líneas investigativas trazadas por el MINSAP dejen de cumplirse y poner empeño en innovar y racionalizar, así como en mantener la continuidad de la docencia y garantizar la renovación de nuevos profesionales capaces de globalizar el sistema de atención al ser humano.

La especialidad de alergología en Cuba ha tenido un salto cualitativo ya que tienen acceso todos los ciudadanos sin tener en cuenta raza, condición social, económica, política o religiosa. Una de sus características es que permite una intervención temprana que puede modificar el curso de la enfermedad. Ha dejado de ser privativa de los que tienen mucho dinero desmitificando tal afirmación. En Cuba la alergología no es una enfermedad de ricos. Está al alcance de todos.

\section{REFERENCI AS BI BLI OGRÁFICAS}

1. J ohansson SGO, Haahtela T. World Allergy Organization Guidelines for Prevention of Allergy and Asthma. Allergy Clin Immunol Int-J World Allergy Org. 2004; 16(5): 176-85.

2. Shearer WT, Huston DP. The immune system. An overview. In: Middleton E, Reed CE, Ellis EF, Adkinson NF, Yunginger JW, Busse W W, editors. Allergy Principles and Practice. 5th eds. St. Louis: Mosby; 1993. p. 3-31.

3. Evans R. Epidemiology and Natural history of asthma, allergic rhinitis and atopic dermatitis. In: Middleton E, Reed CE, Ellis EF, Adkinson NF, Yunginger JW, Busse WW, editors. Allergy Principles and Practice. St. Louis: Mosby; 1993. p. 1111.

4. Adams DC, Saxon A. Immediate hypersensitivity approach to diagnosis. In: Lawlor GJ, Fischer TJ, Adelman DC, editors. Manual of Allegy and Immunology. 3th ed.

Boston: Little Brown and Company; 1994. p. 18-39.

5. Johannes R. History of Anaphylaxis. Allergy and Clin Immunol. 2003; 15(4): 144-7.

6. Ministerio de Salud Pública de Cuba. Grupo Nacional de Alergia. Programa de Desarrollo 2000. Alergología. La Habana: Editorial Ciencias Médicas; 1988.

7. Galindo Santana B. Homenaje al 200 aniversario de la introducción de la vacuna de la viruela en América. Rev Cubana Med Trop. 2004;56(3): 61-2. 


\section{Revista Cubana de Salud Pública. 2011;37(3):338-348}

8. Cohen SG, Evans R. Allergen Immunotherapy in historical perspective. In: Lockley RF, Bukantz SC, editors. Allergen Immunotherapy. 10th ed. New York: Dekker; 1991. p. 1-4.

9. Rojas Ochoa F. Una política de principios. Rev Cubana Salud Pública [Internet]. Mar [citado 4 Feb 2010 ]; 34(1). Disponible en:

http://scielo. sld.cu/scielo.php?script=sci arttext\&pid=S0864-

$\underline{34662008000100001 \& \operatorname{lng}=e s \& n r m=i s o \& t \operatorname{lng}=e s}$

10. López Pardo CM. Conceptualización y valoración de la equidad en salud. Rev Cubana Salud Pública [Internet]. 2007 Sep [citado 4 Feb 2010 ];33(3). Disponible en: http://scielo.sld.cu/scielo.php?script=sci_arttext\&pid=S0864 34662007000300005\&lng=es

11. Castro F. La crisis económica y social del mundo. Informe de la VII Cumbre de Países No Alineados. La Habana: Oficina Publicaciones del Consejo de Estado; 1983.

12. Selman Housein Abdo E. Guía para la Excelencia en la atención médica. La Habana: Editorial Científico Técnica; 2002.

13. Bousquet J, Lockley R, Malling HJ . Allergen Immunotherapy: Therapeutic vaccines for allergic disease. A WHO Position Paper. J Allergy Clin Immunol. 1998; 102: 558-62.

14. Álvarez Castaño LS. El Derecho a la salud en Colombia: una propuesta para su fundamento moral. Rev Panam Salud Pública. 2005; 18(2):129-35.

15. Gosepath S. Consideraciones sobre las fundamentaciones de los Derechos Humanos sociales. En: Alonso MA, Giraldo J, editores. Ciudadanía y Derechos Humanos Sociales. Medellín: Escuela Nacional Sindical; 2001. p.15-58.

16. The WAO/IAACI Meeting: The Primary Prevention of Allergy and Asthma. Allergy Clin Immunol. 2000; 12(6): 288.

17. Wong G, Leung TF, Fok TF. Asthma Epidemiology and Hygiene Hypothesis in Asi. Allergy Clin Immunolol Int. 2004;16(4):155-60.

18. Compalati E, Penagos M, Henley K, Canonica GW. Allergy Prevalence Survey by the World Allergy Organization. Allegy Clin Immunol Int J World Allergy Org. 2007; 19(3): 82-90.

19. Proyecciones de Salud Pública para el año 2015 [Internet]. Ministerio de Salud Pública. La Habana: DNE, MINSAP; 2004 [citado 4 Feb 2010]. Disponible en: http://salud2015.sld.cu/proyecciones-para-el-2015-1.pdf/download

20. Arocha Mariño C, Castillo Guzmán A. Experiencia cubana en la organización de los servicios de salud, una alternativa ante la privatización. Rev Cubana Salud Pública [Internet]. 2007 Mar [citado 2010 Feb 04]; 33(1). Disponible en: http://scielo.sld.cu/scielo.php?script=sci_arttext\&pid=S0864$\underline{34662007000100016 \& \operatorname{lng}=\mathrm{es}}$

21. Rodríguez O, Benítez M, Labrada, J ove V. Ensayo clínico diagnóstico con extracto alergénico de Blomia tropicalis en adultos alérgicos y voluntarios sanos. Revista Alergia Méx. 2000; XLVII(2):57-9.

http://scielo.sld.cu 


\section{Revista Cubana de Salud Pública. 2011;37(3):338-348}

22. Castro RL, Mateo M, Naranjo RM, Navarro B, Álvarez M, Ronquillo M, et al. Correlation between skin tests to Dermatophagoides pteronyssinus,

Dermatophagoides siboney and Blomia tropicalis in Cuban asthmatics. Allergol Immunopathol. 2006; 34(1):23-6.

23. Cartaya J, Orta SD, Rodríguez J, Torres O, Labrada A. Amparo normativo para las vacunas terapéuticas en Cuba. Evaluación del desarrollo clínico farmacológico en los últimos 7 años. Anuario Científico. CECMED. 2005; 2:43-56.

24. Lastre M, Pérez O, Labrada A. (Institute Finlay and BIOCEN) inventors. Antiallergic pharmaceutical composition and methods for the preparing thereof. Cuba patent. No. 22983: 2004.

25. Centro Nacional de Información de Ciencias Médicas. Primera graduación de alergólogos. Información corriente. La Habana: CNICM; 1973.

26. Fernández de Castro Peredo $\mathrm{H}$. Ética Médica en la literatura del siglo XIX. Gac Méd Mex. 2005; 141(4):323-34.

27. González Menéndez R. Humanismo y gestión de salud: pasado, presente y futuro. Rev Cubana Salud Pública [Internet]. Dic 2006 [citado 2010 Feb 04]; 32(4):

Disponible en: http://scielo.sld.cu/scielo.php?script=sci_arttext\&pid=S0864$\underline{34662006000400006 \& \operatorname{lng}=\mathrm{es}}$

28. Borroto Cruz R, Aneiros R. La comunicación humana y la calidad de la atención médica. En: Sariego Sarmientos JA, editor científico. Bioética desde una perspectiva cubana. 2da ed. La Habana: Editorial Félix Varela; 1997. p. 199-206

29. Nuñez J over J. La Ciencia y la Tecnología como procesos sociales. Lo que la educación científica no debería olvidar [Internet]. La Habana: Editorial Félix Varela; 1999 [citado 4 Feb 2010 ]; Disponible en:

http:// www.inder.cu/indernet/Provincias/hlg/documetos/textos/P\% 20S\%20DE\% 20LA \% 20CIENCI A\% 20Y\% 20LA \% 20TECNOLOGIA/P\% 20S\% 20DE\% 20LA\% 20CIENCIA\% 20Y $\% 20$ LA\% 20TECNOLOGIA.pdf

30. Bravo Matarazo E. Bioética de una perspectiva cubana. 2da ed. La Habana: Editorial Félix Varela; 1997.

Recibido: 20 de abril de 2010.

Aprobado: 18 de enero de 2011.

Magaly del Pilar Olivares Elegia. Espadero No. 215 La Víbora 10/10. La Habana 10500. Cuba. Telef.:641 2341. Correo electrónico: magalysolivera@infomed.sld.cu 